\title{
Management of adults with diabetes on the haemodialysis unit: summary of new guidance from the Joint British Diabetes Societies (JBDS) and the Renal Association
}

\author{
WRITING GROUP (AEDITORS): ANDREW FRANKEL,1,A SARA KAZEMPOUR-ARDEBILI,2,A RACHNA BEDI, ${ }^{1}$ \\ TAHSEEN A CHOWDHURY, ${ }^{3}$ PARIJAT DE, ${ }^{4}$ NEVINE EL-SHERBINI, ${ }^{1}$ FRAN GAME, ${ }^{5}$ SARA GRAY, ${ }^{6}$ \\ DAWN HARDY, ${ }^{6}$ JUNE JAMES, ${ }^{7}$ MARIE-FRANCE KONG, ${ }^{7}$ GABBY RAMLAN, ${ }^{8}$ ELIZABETH SOUTHCOTT, ${ }^{9}$ \\ PETER WINOCOUR 10
}

\begin{abstract}
Diabetic nephropathy remains the principal cause of endstage renal failure (ESRF) in the UK, as elsewhere in the developed world, and its prevalence is set to increase. People with diabetes and ESRF on maintenance haemodialysis are a highly vulnerable group, often with complex comorbidities, who are at high risk of adverse cardiovascular outcomes, which is the leading cause of mortality in this population. The management of people with diabetes receiving maintenance haemodialysis is shared between diabetes and renal specialist teams and the primary care team, with input from additional healthcare professionals providing foot care, dietary support and other aspects of multidisciplinary care. In this setting, one specialty may assume that key aspects of care are being provided elsewhere, which can lead to important components of care being overlooked. People with diabetes and ESRF require improved delivery of care to overcome organisational difficulties and barriers to communication between health-

Imperial College Healthcare NHS Trust, London, UK

Endocrinology \& Metabolism Research Institute, Tehran University of Medical Sciences, Iran

The Royal London Hospital, Whitechapel, London, UK

${ }_{4}$ Birmingham City Hospital (Sandwell and West Birmingham Hospitals NHS Trust), Birmingham, UK

Derby Teaching Hospitals NHS Foundation Trust and University of Nottingham, UK

East \& North Herts NHS Trust, UK

University Hospitals of Leicester NHS Trust, UK

8 North Middlesex University Hospital NHS Trust, UK

St James University Hospital, Leeds, UK

${ }^{10}$ Queen Elizabeth II Hospital, Welwyn Garden City, UK
\end{abstract}

Address for correspondence: Dr Andrew Frankel Imperial College Healthcare NHS Trust, London, UK. Tel: +44 (0)2083835171

E-mail: a.frankel@imperial.ac.uk

http://dx.doi.org/10.15277/bjd.2016.073 care teams. No comprehensive guidance on the management of this population has previously been produced. These national guidelines, the first in this area, bring together in one document the disparate needs of the diabetes patient on maintenance haemodialysis. The guideline is based on the best available evidence, or on expert opinion where there is no clear evidence to inform practice. We aim to provide clear advice to clinicians caring for this vulnerable population and to encourage and improve education for clinicians and patients to promote patient empowerment and self-management.

Br J Diabetes 2016;16:69-77

Key words: diabetes, end-stage renal failure, chronic kidney disease, haemodialysis, guideline

Why we need this guideline

Diabetic nephropathy remains the principal cause of end-stage renal failure (ESRF) in the UK, ${ }^{1}$ as elsewhere in the developed world, ${ }^{2}$ and the well-described projected global increase in the prevalence of diabetes implies an increasing burden of this disease. People with diabetes on regular hospital haemodialysis are a vulnerable group at high risk of adverse cardiovascular outcomes, the leading cause of mortality in this population. ${ }^{3}$

Accordingly, people with diabetes and ESRF require improved delivery of care, in particular to overcome organisational difficulties. These national guidelines, the first in this area, are intended to support the practice of all healthcare professionals who care for this vulnerable group of patients, based on the best available evidence or on expert opinion where there is no clear evidence to inform practice. We aim to provide clear advice to clinicians caring for people with diabetes on maintenance haemodialysis and to encourage and improve education for clinicians and patients to promote patient empowerment and self-management.

An abridged version of the guideline is presented here to provide an overview of the scope of the documents. Abbreviated recommendations are given at the head of each section. The full 


\section{Box 1 Evidence grades for recommendations}

$1 \mathrm{~A}$ Strong recommendation: high-quality evidence

$1 B$ Strong recommendation: moderate-quality evidence

1C Strong recommendation: low-quality evidence

1D Strong recommendation: very low-quality evidence

$2 A$ Weak recommendation: high-quality evidence

$2 B$ Weak recommendation: moderate-quality evidence

2C Weak recommendation: low-quality evidence

2D Weak recommendation: very low-quality evidence

guideline is available online ${ }^{4}$ : consult this before designing a therapeutic intervention. Evidence grades for recommendations are given in Box 1.

\section{Section 1: Organisation of care}

\section{Summary of key recommendations}

- Documented annual review for all (foot, eye screening) with findings available to all healthcare staff caring for the patient (1B).

- Each patient should have access to: a named link worker on the dialysis unit to ensure screening is carried out and acted upon (1B); a named diabetes specialist nurse (ideally within the outpatient clinic) to support education and ongoing care (1D); a link nurse on the renal unit to coordinate foot care and provide education on diabetes self-care (1D).

- Establish process to coordinate management of acute metabolic, eye, cardiovascular or foot emergencies, with effective communication between the specialist dialysis/ diabetes teams and primary care (1C).

- Specialist diabetes input for all patients with glycaemic instability (1C).

The management of these patients is complex, with a strong requirement for effective multidisciplinary care, but guidance on glycaemic targets or management algorithms has been lacking. Ideally, all patients undergoing maintenance haemodialysis should be reviewed in combined renal diabetes clinics, but attendance rates are often low and such clinics are not well established. The low participation rates probably reflect the fact that many of these patients are elderly and socially deprived, with lives dominated by their dialysis schedule. Indeed, important aspects of care may be overlooked, with renal, diabetes and primary care physicians assuming that these are being met elsewhere. ${ }^{5}$ Diabetes specialist nurses should play a vital role in coordinating care and signposting patients for urgent care for eye, foot or acute metabolic complications. They can support, educate and empower such patients and staff; their review of individuals on dialysis units alongside haemodialysis staff would ensure that all individuals have ongoing diabetes support and timely intervention.

\section{Section 2: Assessment of glycaemic control}

\section{Summary of key recommendations}

- Measure glycated haemoglobin $\left(\mathrm{HbA}_{1 \mathrm{c}}\right)$ ideally with a high performance liquid chromatography-based assay to prevent overestimation due to carbamylation of haemoglobin - you need to know the method your laboratory uses (1A).

- Understand the factors that render $\mathrm{HbA}_{1 \mathrm{c}}$ less reliable on maintenance haemodialysis, e.g. recent transfusions, haemaglobinopathy, increased haemoglobin due to erythropoietin (1A); $\mathrm{HbA}_{1 \mathrm{c}}$ will likely underestimate in the presence of good to moderate glycaemic control unless iron deficient, when it can overestimate glycaemic control (1B).

- Glycated albumin measurement should be restricted to research use $(2 \mathrm{C})$.

- Self-monitoring of blood glucose (SMBG) remains a cornerstone of care for patients with diabetes on maintenance haemodialysis at risk of hypoglycaemia (1A); ensure provision of equipment, testing strips and training for optimal SMBG (1B).

- Use of continuous glucose monitoring (CGM) in this population is limited by technical issues relating to calibration; initiate CGM (if used) on a non-dialysis day to avoid calibration problems caused by dialysis-associated fluctuations in blood glucose (1C).

\section{Biomarkers}

$\mathrm{HbA}_{1 \mathrm{c}}$ measurement is the main biomarker for assessing glycaemic control in chronic kidney disease (CKD) patients with diabetes. ${ }^{6}$ However, the accuracy of the $\mathrm{HbA}_{1 c}$ values in late CKD stages may be open to question, 7,8 and the relationship between $\mathrm{HbA}_{1 \mathrm{c}}$ and average glycaemia has not been confirmed in patients receiving dialysis. Haemoglobinopathies may also render high performance liquid chromatography-based measurement of $\mathrm{HbA}_{1 \mathrm{c}}$ unreliable. ${ }^{9}$ Racial and ethnic differences in the relationship between $\mathrm{HbA}_{1 \mathrm{c}}$ and blood glucose may occur. ${ }^{10}$ Anaemia may also distort the relationship between $\mathrm{HbA}_{1 \mathrm{c}}$ and blood glucose.

$\mathrm{HbA}_{1 \mathrm{c}}$ can be overestimated in the setting of increased blood urea nitrogen (due to production of carbamylated haemoglobin, which is read as $\mathrm{HbA}_{1 \mathrm{c}}$ in electrical charge-based assays); uraemia (increased glycosylation rate); and iron deficiency (common in haemodialysis). Factors that cause underestimation of $\mathrm{HbA}_{1 \mathrm{c}}$ in ESRF include uraemia (shortened erythrocyte life); need for blood transfusions; and use of erythropoietin (higher proportion of younger red blood cells). Nevertheless, $\mathrm{HbA}_{1 \mathrm{c}}$ remains of value in these patients and a correction factor has been proposed for use in dialysis patients (Table 1). ${ }^{11}$ Fructosamine or glycated albumin levels are unaffected by the factors listed above, but the use of alternative biomarkers to $\mathrm{HbA}_{1 \mathrm{c}}$ outside the research setting is insufficiently validated at this time and they may be unreliable if there is hypoalbuminaemia. 
Table 1 Correction factor for glycated haemoglobin $\left(\mathrm{HbA}_{1 \mathrm{c}}\right)$ measurement in patients on dialysis 11

\begin{tabular}{lll}
\hline Haematocrit & $\begin{array}{l}\text { Treatment } \\
\text { with } \\
\text { erythropoietin }\end{array}$ & $\begin{array}{l}\text { Adjustment } \\
\text { for } \\
\mathrm{HbA}_{1 c}\end{array}$ \\
$\geq 30 \%$ & - & $\mathrm{HbA}_{1 \mathrm{c}} \times 1.14$ \\
$<30 \%$ & Low dosage & $\mathrm{HbA}_{1 \mathrm{c}} \times 1.19$ \\
$<30 \%$ & High dosage & $\mathrm{HbA}_{1 \mathrm{c}} \times 1.38$
\end{tabular}

Self-monitoring of blood glucose and continuous glucose monitoring

Self-monitoring of blood glucose (SMBG) is especially important in subjects receiving treatment(s) that may cause hypoglycaemia, those who suffer from regular hypoglycaemia, or those with hypoglycaemia unawareness. The reliability of SMBG varies with the meter used (this should conform to ISO1519712), frequency/timing of measurements, the age/type of test strips (and the environmental conditions in which they are used), the expertise of the person carrying out the test and clinical factors (e.g. haemolysis, anticoagulation, hyperlipidaemia and metabolic acidosis). SMBG accuracy is instrument- and user-dependent, so it is important to evaluate each patient's technique regularly and ideally validate the recorded results.

Continuous glucose monitoring (CGM) is ideally suited for people with diabetes on maintenance haemodialysis, as it provides information on short-term glucose fluctuations associated with dialysis. ${ }^{13}$ Calibration is an issue in these patients, however, as SMBG readings may be less reliable in people with ESRF, and may be changing rapidly around the time of dialysis. In addition, the time lag between capillary (SMBG) and interstitial glucose (CGM) is increased in ESRF, which reduces the ability of CGM to detect hypoglycaemia. Comparing CGM data between individuals is also difficult due to these uncertainties.

\section{Section 3: Glycaemic control and outcomes on maintenance haemodialysis}

\section{Summary of key recommendations}

- Set an individualised $\mathrm{HbA}_{1 \mathrm{c}}$ target but aim for 58-68 $\mathrm{mmol} / \mathrm{mol}(7.5-8.5 \%)$ where a treatment associated with hypoglycaemia is used; reduce treatment if $\mathrm{HbA}_{1 \mathrm{c}}$ $<58 \mathrm{mmol} / \mathrm{mol}(7.5 \%)$ in such patients (1C).

- $\mathrm{HbA}_{1 \mathrm{c}}>80 \mathrm{mmol} / \mathrm{mol}(9.5 \%)$ indicates poor glycaemic control unless there is severe iron deficiency (2C).

While the long-term clinical benefits from maintaining effective glycaemic control in diabetes before ESRF is established are well known, ${ }^{14,15}$ the benefits of improving chronic hyperglycaemia at the stage of haemodialysis are less clear and avoiding hypoglycaemia remains a priority to optimise outcomes. In the shorter term, attempting to intensively reduce $\mathrm{HbA}_{1 c}$ has little effect on outcomes and has the potential to increase mortality associated with severe hypoglycaemia, including in diabetes patients with CKD. ${ }^{16-19}$ As there are no specific glycaemic guidelines for the ESRF population, this group of patients should be considered to be vulnerable to these outcomes. We therefore recommend individualised glycaemic goals for diabetes patients on maintenance haemodialysis, with less tight targets for those on treatments known to cause hypoglycaemia.

\section{Section 4: Antidiabetic therapies}

\section{Summary of key recommendations}

- Avoid sulfonylureas or metformin in these patients (no indication, and risk of hypoglycaemia/lactic acidosis, respectively) $(1 B)$; acarbose is not indicated for this population; rarely consider cautious use of repaglinide but experience is limited (2C).

- Pioglitazone is not licensed for use in these patients, but there is some evidence for benefit in this population (no dose reduction required) (1C).

- Insufficient experience to support the use of a glucagon-like peptide 1 (GLP-1) agonist (2C).

- DPP4 inhibitors are indicated, with dose reductions needed currently for sitagliptin, vildagliptin and alogliptin but not linagliptin (1B).

- Avoid sodium glucose co-transporter 2 (SGLT2) inhibitors (no indication).

- Use dialysate containing glucose for all with diabetes on glucose-lowering medication (1C).

- Apply insulin to improve quality of life and avoid extreme variations of blood glucose; CGM may be useful here (1D).

- Most benefit from reduced insulin doses on the dialysis day (individualise [1C]; basal-bolus may be best suited to the glycaemic variability typically seen in this population (expert recommendation); consider analogue basal insulin where $\mathrm{NPH}$ causes hypoglycaemia (1C) and for patients less able to manage basal-bolus (1D)

\section{Overview}

As renal function declines, peripheral insulin resistance increases, with declines in renal gluconeogenesis, hypoglycaemic counterregulation and the clearance of insulin (endogenous or injected) and other antihyperglycaemic agents. Some patients on maintenance haemodialysis can stop taking antidiabetic therapies transiently or permanently. ${ }^{20}$ This summary will focus on the pharmacological groups used in the management of type 2 diabetes in haemodialysis patients. Algorithms for treatment of diabetes mellitus in haemodialysis patients should be individualised based on the safety profile and treatments available locally. 


\section{Non-insulin antidiabetic therapies for type 2 diabetes}

Sulfonylureas (SU) do not have clear indications for use in severe CKD. Also, SU are generally highly protein bound and therefore unlikely to be dialysed, which can cause post-dialysis hypoglycaemia. Active metabolites of glibenclamide or glimepiride may accumulate in CKD; these agents are contraindicated in CKD stages $\geq 3$ (estimated glomerular filtration rate (eGFR) $<60 \mathrm{~mL} / \mathrm{min}$ ). Gliclazide poses a lower risk for severe hypoglycaemia than glibenclamide and glimepiride, but should be used with caution when GFR is $<40 \mathrm{~mL} / \mathrm{min}$. Accordingly, SU should be avoided in diabetes patients on maintenance haemodialysis due to their limited therapeutic indications and risk of hypoglycaemia. Repaglinide and nateglinide are highly protein bound and therefore unlikely to be removed in dialysis and should be used with caution in people on haemodialysis.

Metformin has no clinical value in the haemodialysis population due to the risk of severe lactic acidosis secondary to drug accumulation.

Acarbose has not been studied in severe renal impairment. Accordingly, it is not recommended for use in dialysis.

Pioglitazone has a low risk of hypoglycaemia and its effects are not influenced by haemodialysis. It can be used in CKD down to a clearance of $4 \mathrm{~mL} / \mathrm{min}$ without dose adjustment; however, it is not licensed for use in patients on haemodialysis. Thiazolidinedione-associated 'heart failure' is largely caused by salt and water retention; the risk of this in patients on maintenance haemodialysis has not been studied and thus we would not recommend pioglitazone in haemodialysis patients.

Clinical experience with glucagon-like peptide 1 (GLP-1) agonists in patients with renal impairment is limited. Although daily liraglutide and weekly dulaglutide can be used where eGFR is $>50 \mathrm{~mL} / \mathrm{min}$, caution is recommended for use in patients with severe CKD. Lixisenatide and exenatide cannot be recommended for patients with severe CKD, including those on haemodialysis. ${ }^{21}$ Post-marketing reports of worsened renal function with GLP-1 agonists have appeared, especially in patients reporting gastrointestinal side-effects or dehydration. On balance, GLP-1 analogues cannot currently be recommended for patients on haemodialysis.

DPP4 inhibitors can be used in patients on haemodialysis. Within this class, only linagliptin does not need a dose reduction in this setting as it is not eliminated via the kidney. Dose reductions are required for patients on haemodialysis for other available DPP4 inhibitors: labelled maintenance doses in this setting are sitagliptin (25 mg QD), vildagliptin (50 mg QD), alogliptin (6.25 mg QD). These reductions reflect generally a $50-75 \%$ reduction in the daily dose for people without CKD. Saxagliptin is not indicated for use in people with ESRF.
Sodium glucose co-transporter 2 (SGLT2) inhibitors (currently canagliflozin, dapagliflozin and empagliflozin) inhibit glucose re-absorption in functioning proximal renal tubules, thus providing an insulin-independent antihyperglycaemic mechanism. Since their glucose-lowering action requires a working kidney, they are contraindicated in haemodialysis patients.

\section{Insulin in patients with end-stage renal failure}

Insulin requirements fall in line with reductions in eGFR. The process of haemodialysis has a number of effects on glycaemic control. These include removal of glucose (the dialysate should contain glucose for all patients with diabetes on glucose-lowering medication), removal of glucoregulatory hormones (insulin, C-peptide, glucagon) and some antidiabetic drugs (insulin, some $\mathrm{SU})$ and modulation of insulin action secondary to improved uraemia, acidosis and phosphate metabolism. Therefore, glucose control on dialysis days may be very different from that on nondialysis days, leading to unpredictable glucose levels and glycaemic variability. While requirements for antihyperglycaemic therapy may be reduced in these patients to avoid hypoglycaemia (the so-called 'burnt-out diabetes' phenomenon), most patients with diabetes on maintenance haemodialysis require some therapy for hyperglycaemia. Importantly, variation of oral hypoglycaemic or insulin therapy may be required on the day of dialysis when glucose levels will often be lower and glucose variability higher.

A basal-bolus regimen with regular SMBG may be the safest regimen, with analogue insulin considered in place of $\mathrm{NPH}$ where recurrent hypoglycaemia occurs (Figure 1). Alternatively, thriceweekly long-acting insulin at the end of dialysis in diabetic haemodialysis patients may improve glycaemic control significantly (further research on this is needed with new ultra-longacting insulins such as degludec).22,23 Biphasic insulin regimens may be more difficult to manage on haemodialysis due to the irregularity of diet, glucose levels and activity imposed by haemodialysis sessions, but patients stabilised on a biphasic insulin regimen may be reluctant to change. Advice on 10-15\% reduction in doses of insulin on dialysis days may be required to avoid hypoglycaemia. Some patients on biphasic insulin may need a bedtime carbohydrate snack $(<20 \mathrm{~g})$ to reduce the risk of nocturnal/early morning hypoglycaemia.

\section{Section 5: Dietary management and nutrition Overview}

Patients with diabetes who commence maintenance haemodialysis may have received dietary advice from the diabetes and renal team, from dietitians or other health professionals, each with their own priority. This can lead to confusion and ultimately poor dietary adherence. Patients should identify achievable goals and changes to lifestyle behaviours, with good communication between specialties to help reduce confusion and provision of contradictory information. A clear care plan helps to achieve a holistic approach to patient care. It is therefore important that haemodialysis patients are routinely referred to a registered dietitian who is qualified to assess their overall diet and offer 
Figure 1. Management of hyperglycaemia with insulin in the hospital/satellite dialysis unit.

- Patients should be encouraged to monitor and manage their own diabetes as far as possible

- Patients should bring their own insulin/tablets with them to the dialysis unit

- In patients on agents that could cause hypoglycaemia - blood glucose should be checked pre-dialysis and just before finishing dialysis

- Blood glucose can fluctuate during dialysis and most frequently drops in the last hour of dialysis

- Reduce total insulin dose by $10-15 \%$ during and immediately following dialysis

- Reduction in insulin (or OAD) dose is required in those with $\mathrm{HbA}_{1 \mathrm{c}}<58 \mathrm{mmol} / \mathrm{mol}(7.5 \%)$

For a patient maintained on:

\section{Rapid-acting insulin}

Patients should reduce their usual breakfast (if morning dialysis), lunchtime (if afternoon dialysis), or evening insulin (if evening dialysis) by $10-15 \%$ at the start of each shift

\section{Premixed/biphasic insulin}

Patients should reduce dose by $10-15 \%$ with breakfast (morning or afternoon dialysis) and with their evening meal (if starting evening dialysis)
Long-acting insulin

Patients should reduce the dose by $25 \%$ in the morning or evening of dialysis

\section{With blood glucose values of:}

Pre-dialysis $<7 \mathrm{mmol} / \mathrm{L}$

- Give 20-30g carbohydrate prior to dialysis

- Recheck blood glucose before finishing dialysis

- May need a carbohydrate snack before end of dialysis
Pre-dialysis 7-15 mmol/L

- No action required
$>15 \mathrm{mmol} / \mathrm{L}$ just before finishing dialysis

- Ask patient to monitor BMs and seek advice from the general practitioner or Diabetes Specialist Nurse if persistently high

\section{Summary of key recommendations}

- Identify dietary aims according to the type of diabetes (1C) and review nutritional support regularly to minimise glycaemic variability (1D); promote and include behaviour change and increased activity to achieve/maintain healthy weight (1B).

- Each haemodialysis unit should have access to dietary expertise to provide a holistic personalised approach relevant to diabetes and renal care - provide advice to minimise glycaemic excursions on both dialysis and non-dialysis days (1D).

- Aim for energy intake 30-40 kcal/kg ideal body weight (IBW): $50-60 \%$ from carbohydrate, $<30 \%$ from fat, $\geq 15 \%$ from protein, with individualised advice for those aiming to lose weight (1D) and a protein intake of $>1.1 \mathrm{~g} / \mathrm{kg} \mathrm{IBW} \mathrm{(1C).}$

- Encourage consumption of low-potassium fruit, vegetables and carbohydrates with a low to moderate glycaemic index (GI), with '5-a-day' fruit and vegetable portions; focus on foods containing phosphate additives before reducing low-GI foods (1D).

- $\quad$ Salt intake $<6 \mathrm{~g} /$ day $(1 \mathrm{C})$.
- Limit oily fish consumption due to vitamin $\mathrm{A}$ and phosphate content (2D).

- A water-soluble vitamin supplement is recommended (expert recommendation).

- Screen all for protein energy wasting (PEW) on each admission to hospital and establish a procedure at the dialysis unit to identify those at risk of PEW for referral to the dietitian (1D).

- Dietary counselling and oral nutrition support is the first-line treatment for patients unable to meet their nutritional needs orally; parenteral feeding may be necessary if these interventions are insufficient (1D).

- Assess glucose before and after dialysis for insulin-treated patients and give 20-30 g of low-Gl carbohydrate at the beginning of haemodialysis if pre-dialysis glucose is $<7 \mathrm{mmol} / \mathrm{L}$ (1D).

- Ensure patients can access support for hypoglycaemia at all times, including travelling to and from the dialysis unit (expert recommendation). 
- Educate patients and staff on managing mild to moderate hypoglycaemia and hypoglycaemia unawareness; where hypoglycaemia occurs, provide treatment with rapid-acting carbohydrate within fluid, potassium and phosphate restrictions (1D).

- Small, regular, low-fat and low-fibre meals are recommended to manage gastroparesis (1C).
- Ensure that patients understand that they are more likely to achieve inter-dialytic weight gain $<2 \mathrm{~kg}$ if they optimise their $\mathrm{HbA}_{1 \mathrm{c}}(1 \mathrm{C})$.

- Encourage overweight/obese patients considered for kidney transplant to lose weight (calorie-restricted diet with protein $\geq 1.1 \mathrm{~g} / \mathrm{kg}$ IBW) (1B); consider bariatric surgery or weight loss medication if unable to lose sufficient weight for transplantation otherwise (1C). appropriate individualised advice. ${ }^{24}$

The full guideline ${ }^{4}$ provides detailed advice on these aspects of nutritional support, and a highly abbreviated précis is provided here.

\section{Key dietary components}

Energy intake should comprise $30-40 \mathrm{kcal} / \mathrm{kg}$ ideal body weight (50-60\% from carbohydrate, $<30 \%$ from fat and $>15 \%$ from protein). An individualised plan is needed for those seeking to lose weight but to maintain body mass index (BMI) at $\geq 23$ $\mathrm{kg} / \mathrm{m}^{2} .25$ The diabetes team can advise on balancing glycaemic control with increased nutrition support for patients who require an increased calorie load. Protein intake should be $>1.1 \mathrm{~g} / \mathrm{kg}$ ideal weight. Carbohydrate intake should be agreed within overall aims of diabetes management, separately for dialysis and non-dialysis days, with provision of education on insulin dose adjustment and carbohydrate management/counting, where applicable.

Individualised low-potassium dietary advice is indicated if potassium is $\geq 6.0 \mathrm{mmol} / \mathrm{L}$. Consumption of low-potassium carbohydrate options (e.g. pasta, rice, noodles, bread) may support greater intake of fruits and vegetables within a healthier diet overall. Insulin deficiency can promote hyperkalaemia.

Dietary advice should be provided to maintain serum phosphate at 1.1-1.7 mmol/L. Reduce foods containing phosphate additives before reducing low-glycaemic index (Gl) foods, or those high in natural protein (e.g. eggs, nuts, dairy, seafood). Wholegrain products are high in phosphate, but this is unlikely to be absorbed due to their phytate content.

Salt should be limited to an intake of $<6 \mathrm{~g} /$ day.

Provision of a water-soluble vitamin supplement is recommended, although there are no randomised trials to support this. Finally, advise caution regarding intake of oily fish, mainly because of its vitamin A and phosphate content.

\section{Nutrition support}

\section{Prevention of protein energy wasting}

Protein energy wasting (PEW) is a major cause of morbidity and mortality in haemodialysis patients, especially in diabetes. Ensuring adequate energy and protein intake and optimising dialysis prescription is recommended. All inpatients should be screened for PEW on admission and weekly thereafter. Screening tools in hospitals may not be specific enough to identify a dialysis patient at risk of PEW, particularly those using weight and BMI, as fluid changes can complicate their interpretation. Outpatients should be screened at their first clinic appointment and/or at initiation of dialysis and then 3-6-monthly. There should be procedures for referral to the renal dietitian if any member of the team identifies risk of PEW.

\section{Delivering nutrition support}

Nutrition support should be considered in haemodialysis patients with any of the following: $\mathrm{BMl}<20 \mathrm{~kg} / \mathrm{m}^{2}$; unintentional nonoedema weight loss $>5-10 \%$ over $3-6$ months; $<85 \%$ ideal weight; subjective global assessment graded B/C or 1-5; intercurrent catabolic acute conditions preventing normal nutrition or adequate oral intake. All malnourished haemodialysis patients should receive dietary counselling, including advice on the use of oral nutritional supplements (ONS) and non-saturated fats where necessary. ${ }^{26}$ Long-term nasogastric or gastrostomy feeding should be considered where ONS is insufficient. When intensive dietary counselling, ONS and enteral feeding have failed, intra-dialytic parenteral nutrition is recommended. Maintenance of serum glucose $6-10 \mathrm{mmol} / \mathrm{L}$ is recommended to avoid postdialysis hypoglycaemia. Investigate and overcome causes of reduced oral intake.

\section{Managing mild hypoglycaemia}

Dialysis patients are at risk of hypoglycaemia, especially within $24 \mathrm{~h}$ of dialysis (see Section 6 of the full guideline). Intake of 10-20 g of a low-Gl carbohydrate is recommended at the second hour of haemodialysis.

If the pre-haemodialysis blood glucose is $<7 \mathrm{mmol} / \mathrm{L}, 20-30 \mathrm{~g}$ carbohydrates at the beginning of haemodialysis is recommended. For patients with hypoglycaemia symptoms we recommend intake of 15-20 g carbohydrate (e.g. one medium slice of bread or two digestive biscuits). Patients should have access to a remedy for hypoglycaemia at all times, including during travelling to and from the dialysis unit. In patients given a large amount of food on dialysis, there may be an increased incidence of hypotension during hours 3-4 due to increased intestinal blood flow.

Hypoglycaemia in hospital should be managed according to recommendations from the Joint British Diabetes Societies. ${ }^{27}$ Many sources of rapid-acting glucose recommended for treating hypoglycaemia can be inappropriate for maintenance haemodialysis patients due to their potassium, phosphorus or fluid content. 


\section{Managing gastroparesis}

Small, frequent, low-fibre and low-fat meals with increased liquid nutrient intake (including liquid fat where increased caloric intake is needed) may be appropriate for patients with gastroparesis on haemodialysis (no alcohol or carbonated drinks). Foods of small particle size (or pureed) may also be helpful. It is advised to reduce the amount of insoluble fibre in the diet of patients with gastroparesis to prevent phytobezoar accumulation. ${ }^{28}$ For patients with gastroparesis requiring enteral feeding, post-pyloric enteral feeding such as jejunal feed (placed surgically or endoscopically) is appropriate.

\section{Fluid management}

Glycaemic control is important in the fluid management of dialysis patients with diabetes as poor glycaemic control can lead to a vicious cycle of thirst and polydipsia and increases the risk of a higher IDWG (>4.5\% of dry weight).

\section{Managing obesity}

The focus of nutritional care in patients on maintenance haemodialysis should be on total energy intake, rather than the source of energy in the diet, for optimal glycaemic control and weight reduction. However, there are virtually no standards, guidelines or studies regarding obesity in patients with diabetes on haemodialysis.

Some studies suggest that obesity or weight variation are positively correlated with survival of patients on dialysis. ${ }^{29,30} \mathrm{How}$ ever, some authors question the existence of this 'obesity paradox'. There is very limited evidence of the benefits of specific diets or bariatric surgery for weight loss in the CKD population, especially those on maintenance haemodialysis. Pharmacological treatment may be considered for people who have not reached their target weight loss on diet, activity and behavioural changes. ${ }^{31} \mathrm{~A}$ lack of experience with GLP-1 agonists means they cannot presently be recommended in subjects with renal failure.

\section{Section 6: Complications of diabetes in haemodialysis patients}

\section{Summary of key recommendations}

- Be aware that poor/erratic nutritional intake, reduced hepatic/renal insulin clearance and decreased hepatic gluconeogenesis promote hypoglycaemia in this population (1B).

- Counsel patients on hypoglycaemia and hypoglycaemia unawareness (1B), especially on dialysis days (consider reducing anti-hyperglycaemic therapy on dialysis days [1D]).

- These patients require regular podiatry review with formal 3-monthly screening by competent staff on the dialysis unit using a locally agreed tool (1C).

- Refer patients with an ulcer or other concern to the diabetic foot multidisciplinary team within one working day, and refer a patient with a hot swollen foot within $24 \mathrm{~h}$ (1D).
- Use a pressure-relieving device on heels during dialysis; inspect feet at least weekly (expert recommendation).

- The clinician in charge of care (nephrologist or diabetologist) should ensure that the patient on home dialysis attends an annual review by the foot protection team (1D).

- Patients approaching end of life or under palliative care should be managed in accordance with Diabetes UK End of Life clinical care recommendations (1D).

\section{Glycaemic variability and hypoglycaemia}

Reduced insulin clearance in advanced CKD leads to lower insulin requirements and a higher risk of hypoglycaemia if insulin or SU are not reduced. Uraemia-induced reduction in calorie intake may also reduce insulin requirement. Hypoglycaemia appears to occur twice as frequently amongst patients with CKD as in those with normal renal function, ${ }^{32}$ usually in the hours after dialysis in patients with ESRF. Hypoglycaemic unawareness is common in these patients.

Haemodialysed subjects usually have their lowest glucose readings after dialysis, and a dialysate containing glucose should be used for all patients with diabetes on glucose-lowering medication. Clinicians may need to identify subjects' individual glycaemic profile patterns and vary antidiabetic treatment accordingly. ${ }^{33}$ Patients with diabetes with similar $\mathrm{HbA}_{1 \mathrm{c}}$ may have significantly different daily plasma glucose profiles within or between days. Long-term glycaemic variability increases the hypoglycaemia risk and may promote vascular complications of diabetes. ${ }^{34-38}$ However, there is limited evidence for addressing glycaemic variability as a strategy for improving cardiovascular outcomes. Further research is needed to define the role of CGM and optimal insulin administration in the dialysis setting (see above).

\section{Diabetic foot disease in renal dialysis patients}

ESRF and CKD 4-5 are independent risk factors for diabetic foot disease, neuropathy, peripheral arterial disease, delayed wound healing, amputation and post-amputation mortality. Dialysis/ renal replacement therapy is strongly and independently associated with foot ulcers. ${ }^{39-42}$ Neuropathy greatly increases the risk of pressurerelated ulcers, and care must be taken to ensure adequate pressure relief on patients' heels in renal dialysis units when they are recumbent for prolonged periods. ${ }^{43}$ Podiatry input on dialysis units reduces the frequency and severity of diabetic foot complications: regular podiatry assessment (at least 3-monthly) should be ensured, ideally on dialysis units as this frail, multi-morbid population may have difficulty accessing community podiatry services.

The Charcot foot (Charcot neuropathic osteoarthropathy) is associated with renal disease and very high morbidity. It is frequently misdiagnosed as infection, venous thrombosis or gout, leading to potentially avoidable limb loss. A non-removable cast or walker is recommended for offloading an acute Charcot foot. However, patients on renal replacement therapy may tolerate this poorly due to changing peripheral oedema, and other methods of offloading (e.g. removable cast and wheelchair use) may be required. 


\section{Key messages}

- The number of patients on maintenance haemodialysis who also have diabetes is increasing progressively in the UK and constitute a significant proportion of the maintenance haemodialysis population

- Management of these patients is complex and there is limited evidence to support optimal glycaemic targeting and management

- Management should be aimed at mitigating complications of diabetes which particularly relate to hypoglycaemia and vascular disease

- In order to best achieve these aims, there needs to be appropriate organisational arrangements based around the patient's needs

\section{End of life care in patients with diabetes on maintenance haemodialysis}

A decision to withdraw from renal replacement therapy is recognised as a common cause of death. Clear guidance for the management of end of life care in these patients is essential in order to support teams (including palliative care) and carers during this difficult time. ${ }^{44}$ Diabetes adds to the complexity of care planning for end of life and early liaison with the diabetes specialist team is recommended. Teams need to ensure that the patients' wishes are paramount when planning end of life care and that effective communication with the patient, their relatives or carer and primary care physician is in place.

Diabetes medications, including insulin, may be reduced or stopped in some people with type 2 diabetes to avoid hypoglycaemia (but this can lead to diabetic ketoacidosis (DKA) and severe dehydration in people with type 1 diabetes). Glucose monitoring can be minimised to once/day (glycaemic target 6-15 $\mathrm{mmol} / \mathrm{L}$ without diabetes symptoms) in those receiving insulin treatments and is only used to rule out hypoglycaemia, hyperosmolar hyperglycaemic state or DKA. The giving of fluids is entirely the choice of the patient or carer.

Acknowledgements A medical writer (Dr Mike Gwilt, GT Communications) provided editorial assistance (free of charge) in abridging the original guideline to produce this article. Versions of this article will appear in both the British Journal of Diabetes and Diabetic Medicine, reflecting funding of JBDS by the Association of British Clinical Diabetologists and Diabetes UK, respectively. Conflict of interest AF: Preparation of educational materials (BoehringerIngleheim/Lilly Alliance, AstraZeneca, Merck, Janssen) and receipt of research grant (Boehringer-Ingleheim); PD: presentation of educational materials (Novo Nordisk, Sanofi, Novartis, Roche, Astra, Lilly Boehringer, MSD, Janssen, Internis, Menarini, Abbott); Jj: preparation and delivery of Educational Materials for Sanofi, Lilly, Novo Nordisk, AstraZeneca, MSD, Janssen. All other co-authors declared no conflicts of interest.

Funding None.

\section{References}

1. UK Renal Registry. 2014 - The Seventeenth Annual Report. Available at https://www.renalreg.org/reports/2014-seventeenth-annual-report/ (accessed Feb 2016).

2. United States Renal Data System. 2012 Atlas of CKD \& ESRD. Available at http://www.usrds.org/atlas12.aspx (accessed Feb 2016).

3. Coresh J, Selvin E, Stevens LA, et al. Prevalence of chronic kidney disease in the United States. JAMA 2007;298:2038-47. http://dx.doi.org/10.1001/jama.298.17.2038

4. Joint British Diabetes Societies and Renal Association. Management of adults with diabetes on the haemodialysis unit. http://www. diabetologists-abcd.org.uk/jbds/jbds.htm

5. Atherton $\mathrm{G}$. Renal replacement and diabetes care: the role of a specialist nurse. J Diabetes Nursing 2004;8:70-2.

6. National Kidney Foundation. KDOQI Clinical Practice Guideline for Diabetes and CKD: 2012 Update. Am J Kidney Dis 2012;60:850-86. http://dx.doi.org/10.1053/j.ajkd.2012.07.005

7. Shima K, Chujo K, Yamada M, et al. Lower value of glycated haemoglobin relative to glycaemic control in diabetic patients with end-stage renal disease not on haemodialysis. Ann Clin Biochem 2012;49:68-74. http://dx.doi.org/10.1258/acb.2011.011161

8. Ansari A, Goldsmith D, Krimholtz M, et al. Measuring glycaemic control in renal failure. Diabet Med 2001;18:70A (abstract).

9. Gunton JE, McElduff A. Hemoglobinopathies and $\mathrm{HbA}(1 \mathrm{c})$ measurement. Diabetes Care 2000;23:1197-8. http://dx.doi.org/10.2337/diacare.23.8.1197

10. Herman WH, Cohen RM. Racial and ethnic differences in the relationship between $\mathrm{HbA} 1 \mathrm{c}$ and blood glucose: implications for the diagnosis of diabetes. J Clin Endocrinol Metab 2012;97:1067-72. http://dx.doi.org/10.1210/jc.2011-1894

11. Uzu T, Hatta T, Deji N, et al. Target for glycemic control in type 2 diabetic patients on hemodialysis: effects of anemia and erythropoietin injection on hemoglobin A(1c). Ther Apher Dial 2009;13:89-94. http://dx.doi.org/10.1111/j.1744-9987.2009.00661.x

12. Diabetes UK. ISO Standards for Blood Glucose Meters. Available at http://www. diabetes.co.uk/blood-glucose-meters/iso-accuracy-standards.html (accessed February 2016).

13. Riveline JP, Teynie J, Belmouaz S, et al. Glycaemic control in type 2 diabetic patients on chronic haemodialysis: use of a continuous glucose monitoring system. Nephrol Dial Transplant 2009;24:2866-71. http://dx.doi.org/10.1093/ndt/gfp181

14. Holman RR, Paul SK, Bethel MA, et al. 10-year follow-up of intensive glucose control in type 2 diabetes. N Engl J Med 2008;359:1577-89. http://dx.doi.org/10.1056/NEJMoa0806470

15. Diabetes Control and Complications Trial/Epidemiology of Diabetes Interventions and Complications (DCCT/EDIC) Study Research Group. Intensive diabetes treatment and cardiovascular disease in patients with type 1 diabetes. N Engl J Med 2005;353:2643-53. http://dx.doi.org/10.1056/NEJMoa052187

16. Action to Control Cardiovascular Risk in Diabetes Study Group. Effects of intensive glucose lowering in type 2 diabetes. N Engl J Med 2008; 358:2545-59. http://dx.doi.org/10.1056/NEJMoa0802743

17. VADT Investigators. Glucose control and vascular complications in veterans with type 2 diabetes. N Engl J Med 2009;360:129-39. http://dx.doi.org/10.1056/NEJMoa0808431

18. ADVANCE Collaborative Group. Intensive blood glucose control and vascular outcomes in patients with type 2 diabetes. N Engl J Med 2008;358:2560-72. http://dx.doi.org/10.1056/NEJMoa0802987

19. ACCORD Study Group. Chronic kidney disease and intensive glycemic control increase cardiovascular risk in patients with type 2 diabetes. Kidney Int 2015;87:649-59. http://dx.doi.org/10.1038/ki.2014.296

20. Park J, Lertdumrongluk P, Molnar MZ, et al. Glycemic control in diabetic dialysis patients and the burnt-out diabetes phenomenon. Curr Diab Rep 2012;12:432-9. http://dx.doi.org/10.1007/s11892-012-0286-3

21. Fonseca VA, Alvarado-Ruiz $R$, Raccah $D$, et al. Efficacy and safety of the once-daily GLP-1 receptor agonist lixisenatide in monotherapy: a randomized, double-blind, placebo-controlled trial in patients with type 2 diabetes (GetGoal-Mono). Diabetes Care 2012;35:1225-31. http://dx.doi.org/10.2337/dc11-1935 
22. Bouchi R, Babazono T, Onuki T, et al. Administration of insulin glargine thrice weekly by medical staff at a dialysis unit: a new insulin regimen for diabetic management in physically impaired patients undergoing hemodialysis. Diabetology Int 2011;2:197-201. http://dx.doi.org/10.1007/s13340-011-0044-9

23. Shoji T, Emoto M, Mori K, et al. Thrice-weekly insulin injection with nurse's support for diabetic hemodialysis patients having difficulty with self injection. Osaka City Med J 2012;58:35-8.

24. Willingham $\mathrm{F}$. The dietary management of patients with diabetes and renal disease: challenges and practicalities. J Renal Care 2012;38(Suppl 1):40-51. http://dx.doi.org/10.1111/j.1755-6686.2012.00283.x

25. Fissell RB, Bragg-Gresham JL, Gillespie BW, et al. International variations in vitamin prescription and association with mortality in the Dialysis Outcomes and Practice Patterns Study (DOPPS). Am J Kidney Dis 2004:44:293-9. http://dx.doi.org/10.1053/j.ajkd.2004.04.047

26 . Fouque $D$, Vennegoor $M$, ter Wee $P$, et al. European best practice guideline on nutrition. Nephrol Dial Transplant 2007;22(Suppl 2):ii45-ii87. http://dx.doi.org/10.1093/ndt/gfm020

27. Joint British Diabetes Societies, Diabetes UK. The Hospital Management of Hypoglycaemia in Adults with Diabetes Mellitus. March 2010. Available at http://www.diabetologists-abcd.org.uk/JBDS/JBDS_IP_ Hypo_Adults.pdf (accessed Feb 2016).

28. Rider JA, Foresti-Lorente RF, Garrido J, et al. Gastric bezoars: treatment and prevention. Am J Gastroenterol 1984;79:357-9.

29. Stolic R. Obesity in renal failure - health or disease? Med Hypotheses 2010;75:497-500. http://dx.doi.org/10.1016/j.mehy.2010.07.004

30. Chazot C, Gassia JP, Di Benedetto A, et al. Is there any survival advantage of obesity in southern European haemodialysis patients? Nephrol Dial Transplant 2009:24:2871-6. http://dx.doi.org/10.1093/ndt/gfp168

31. National Institute for Health and Care Excellence (NICE). Public Health Draft Guidance. Managing overweight and obesity in adults - lifestyle weight management services. Available at https://www.nice.org.uk/guidance/ph53/documents/overweight-and-obese-adults-lifestyle-weightmanagement-draft-guidance2 (accessed Feb 2016).

32. Moen MF, Zhan M, Hsu VD, LD et al. Frequency of hypoglycaemia and its significance in chronic kidney disease. Clin J Am Soc Nephrol 2009;4:1121-7. http://dx.doi.org/10.2215/CJN.00800209

33. Kazempour-Ardebili S, Lecamwasam VL, Dassanyake T, et al. Assessing glycemic control in maintenance hemodialysis patients with type 2 diabetes. Diabetes Care 2009;32:1137-42.

http://dx.doi.org/10.2337/dc08-1688
34. Su G, Mi S, Tao H, et al. Association of glycemic variability and the presence and severity of coronary artery disease in patients with type 2 diabetes. Cardiovasc Diabetol 2011;10:19. http://dx.doi.org/10.1186/1475-2840-10-19

35. Esposito K, Ciotola M, Carleo D, et al. Post meal glucose peaks at home associate with carotid intima media thickness in type 2 diabetes. J Clin Endocrinol Metab 2008;93:1345-50. http://dx.doi.org/10.1210/jc.2007-2000

36. Cavalot F, Petrelli A, Traversa M. Postprandial blood glucose is a stronger predictor of cardiovascular events than fasting glucose in type 2 diabetes mellitus, particularly in women: lessons from the San Luigi Gonzaga Diabetes Study. J Clin Endocrinol Metab 2006;91:813-19. http://dx.doi.org/10.1210/jc.2005-1005

37. Muggeo $M$, Verlato $G$, Bonora $E$, et al. Long term instability of fasting plasma glucose, a novel predictor of cardiovascular mortality in elderly patients with non-insulin dependent diabetes mellitus: the Verona Diabetes Study. Circulation 1997:96:1750-4. http://dx.doi.org/10.1161/01.CIR.96.6.1750

38. Hanefeld $\mathrm{M}$, Cagatay $\mathrm{M}$, Petrowitsch $\mathrm{T}$, et al. Acarbose reduces the risk for myocardial infarction in type 2 diabetic patients: meta-analysis of seven long term studies. Eur Heart J 2004:25:10-16. http://dx.doi.org/10.1016/S0195-668X(03)00468-8

39. Margolis DJ, Hofstad O, Feldman HI. Association between renal failure and foot ulcer or lower-extremity amputation in patients with diabetes. Diabetes Care 2008;31:1331-6. http://dx.doi.org/10.2337/dc07-2244

40. Ndip A, Rutter MK, Vileikyte $L$, et al. Dialysis treatment is an independent risk factor for foot ulceration in patients with diabetes and stage 4 or 5 chronic kidney disease. Diabetes Care 2010;33:1811-16. http://dx.doi.org/10.2337/dc10-0255

41. Game F. Preventing amputations in patients with diabetes and renal disease. Practical Diabetes 2012:29:324-8.

42. Game FL, Chipchase SY, Hubbard R, et al. Temporal association between the incidence of foot ulceration and the start of dialysis in diabetes mellitus. Nephrol Dial Transplant 2006;21:3207-10. http://dx.doi.org/10.1093/ndt/gfl427

43. Ndip A, Lavery LA, Lafontaine J, et al. High levels of foot ulceration and amputation risk in a multiracial cohort of diabetic patients on dialysis therapy. Diabetes Care 2010:33:878-80. http://dx.doi.org/10.2337/dc09-2007

44. Diabetes UK. End of Life Care (October 2013). Available at https://www.diabetes.org.uk/end-of-life-care (accessed Feb 2016). 